\title{
Die Verwendung von Bindemitteln bei der Wurstfabrikation.
}

\author{
Von \\ A. Behre. \\ Mitteilung aus dem Chemischen Untersuchungsamte der Stadt Chemnitz.
}

Mit den Handelsbräuchen ist es im Fleischereigewerbe ein seltsam Ding. Niemand hat sie zwar vorher gekannt, aber plötzlich sind sie da und führen nun ein unbekümmertes Leben. Einige Beispiele mögen das kurz erläutern. Das Hackfleisch und die Wurst müssen bekanntermaßen oft dulden, daß altem Branche entsprechend alle möglichen Abfälle des Fleischereibetriebes in sie hineinverarbeitet werden. Das Aufblasen der Kälber ist als ein alter Brauch im. Fleischergewerbe bezeichnet worden, Ein solcher ist aber in keiner Weise bewiesen, und strenge Verbote haben dann gezeigt, dab man nicht weniger gut auch ohne diese Unsitte auskommen kann. Der Verwendung von Konservierungsmitteln war ja glücklich durch die Verordnung vom 18. Februar 1902 ein Riegel vorgeschoben worden und man wähnte insbesondere die schweflige Säure schon fast vergessen, als sie plötzlich in der Form von Scheuersand als Phönix aus der Asche ihre Auferstehung feierte. Ahnlich ging es mit der Borsäure, die sich seit einiger Zeit in der Hand des Fleischers in Benzoesäure umgewandelt hat. Man sieht deutlich, welche Lebensfähigkeit solche Sitten und Gewohnheiten besitzen und wie schwer sie auszutreiben sind.

Auf dem Gebiete der Wurstbindemittel ist es nicht besser gegangen. Der Fleischer mule doch früher an Mehl und. Semmel keine solche Frende gehabt haben, wie er uns heute glauben machen will, denn Berliner und Leipziger Metzger sollen in den 80-er Jahren des verflossenen Jahrhunderts ausgesagt haben ${ }^{1}$ ), daß der Meblzusatz zu Würstchen seit etwa 10 Jahren Brauch sei und dab er aus Süddeutschland herstamme. Aber auch in Süddeutschland soll der Brauch damals 10-15 Jabre bestanden haben. Natürlich ist der Bindemittelzusatz seitdem in allen Gegenden des deutschen Reiches „Handelsbrauch" und damit scheinbar auch "Gewohnheitsrecht" geworden. Tatsache ist jedenfall;, daß nach Einführung des Nahrungsmittelgesetzes im Jahre 1879 man erst gegen die Verwendung des Mehles im Fleischereigewerbe vorgegangen ist und die Gerichte, insbesondere das Reichsgericht, haben wiederholt Gelegenheit gehabt, sich mit dieser Frage eingehend zu beschäftigen. So hat es in seinen Urteilssprüchen vom 4. Oktober 1883, vom 23. September 1886 und vom 7. Januar 1887 entschieden, daß Mehlzusatz zu Wurst kein allgemeiner Geschäftsgebrauch und daher strafbar sei; es hat dann dieses Urteil am 14. Oktober 1904 bestätigt. Am 3. Dezember 1894 hat es dahin erkannt, daB ein solcher Zusatz zu Cervelatwürsten stets eine Verfälschung in sich scbließe. Immerhin hat das Reichsgericht aber zugegeben, daß, falls für den Ort der Tat ein diesbezüglicher Gebrauch oder eine Gewohnheit bestehe, dann eine strafbare Handlung nicht vorliege (Entscheidung vom 21. Dezember 1882 und 4. Oktober 1883). So sei der Zusatz von Semmeln zu Rostbratwürsten keine Verfälschung. Es hat sich somit die Frage des Mehlzusatzes dahin zugespitzt, ob für den Ort der Tat solche Handelsbräuche bestehen, und solche sind in vielen Teilen des Reiches durch Ge-

$\left.{ }^{1}\right)$ Ostertag, Handbuch der Fleischbeschan 1902, S. 790. 
richtsentscheidungen festgestellt worden, so z. B. in Frankfurt, desgleichen in Württemberg, Rheinland, Westfalen, Brandenburg und Hamburg, wo ein Zusatz von $2 \%$ als zulässig erklärt wurde, während dagegen in Magdeburg, Coblenz, Wiesbaden, Dresten und auch in Chemnitz ein solcher Zusatz als nicht, ortsüblich und daher unzulässig: erklärt worden ist. Auch das Reichsgesundheitsamt hat zu dieser Frage Stellung genommen und einen Zusatz von $2 \%$ Mehl für zulässig erklärt. Dementsprechend sind von Städten oder Bexirken Polizeiverordnungen erlassen worden, welche den Verkehr mit Wurstwaren regelten, insbesondere aber Grenzen über den Mehlgehalt aufstellten. Das Reichsgericht und Kammergericht in Preußen haben die Frage erörtert, ob solche Polizeiverordnungen rechtsgültig seien. Das Reichsgericht hat unter dem 16. Februar 1903 erkannt, daß es vollkommen im Einklang mit dem Nahrungsmittelgesetz stehe, wenn ein Ortsstatut vorschreibe, daß jeder Mehlzusatz dem Käufer kundgegebers werden müsse, und wenn es einen Mehlzusatz von mehr als $4 \%$ verbiete. Dagegen hat das Kammergericht in seinem Urteil vom 16. Januar 1902 entschieden, laß, da das Reichsgesetz die vorliegende Materie nicht erschöpfend behandle, zwar landesrechtliche Strafrorschriften zulässig wären (in Preußen auf Grund des $\$ 6 \mathrm{c}$ des Gesetzes vom 11. März 1850), aber solche Verordnungen dürften einerseits nicht Zusätze gestatten, welche im Sinne des Reichsgesetzes von 1879 und des $\S 367$ No. 7 des R.-St.-G.-B. als Fälschung anzusehen seien; ebensowenig seien sie berechtigt, in maßgebender Auslegung der erwähnten reichsgesetzlichen Bestimmungen festzustellen, welche Zusätze als Verfälschungen der betreffenden Nahrungsmittel erachtet werden sollen und welche nicht. Im allgemeinen sei jeder Zusatz von Mehl zu Wurst als Verfälschung zu betrachten, falls der Zusatz nicht einem Ortsgebrauch entspräche und dem Publikum deklariert sei. Die Polizei-Verordnung sei ungültig, soweit sie den Meblzusatz bei bestimmten Würsten ganz, bei anderen in einem bestimmten Prozentsatze verbiete. Diese Urteile des Reichsgerichts und Kammergerichts widersprechen sich also erbeblich. Gleichviel bestehen und entstehen, zumal natürlich außerhalb Preußens, noch überall Polizeiverordnungen über diesen Gegenstand, die meist einen Mehlzusatz von $2 \%$ als zulässig erklären. Das Kammergericht hat anch Gelegenheit gehabt, sich über den Begriff der Wurst zu äußern und hat diesen im Jahre 1902 dahin definiert, daß Wurst stets nur ein Gemisch von Fleisch, Fett und Gewürzen sei. Dagegen hat der Deutsche Fleischertag vom Jahre 1904 Einwendungen erhoben und folgenden Antrag angenommen: "Wurst ist nicht nur ein Nahrungs-, sondern auch ein Genußmittel, das den Anforderungen, die an seine Schmackhaftigkeit und Bekömmlichkeit gestellt werden, in erster Linie entsprechen muß. Aufgabe der Polizei und Rechtsprechung kann und darf es nicht sein, in die ortsübliche, den Wünschen des konsumierenden Publikums zusagende Herstellung der Wurst beschränkend einzugreifen; ihre Aufgabe kann nur in der Verhinderung unsolider oder betrügerischer Manipulationen erblickt werden." ${ }^{1}$ ) Es liegt jedoch keine Veranlassung vor, von der Auffassung der Gerichte über den Begriff der Wurst abzugehen, zumal im anderen Falle der Willkür Tür und Tor geöffnet wäre.

$\mathrm{Es}$ ist sehr wichtig, hier vorerst darauf hinzuweisen, daß man inbezug auf die Verwendung von Mehl zur Wurstfabrikation zweierlei Würste zu unterscheiden hat, nämlich Dauerwürste und Brühwürste. Bei den ersteren ist Mehlzusatz un bedingt als Verfälschung zu betrachten, wie nicht allein aus Gerichtsentscheidungen, sondern auch aus den wiederholten Äußerungen des Fleischerverbandes selbst hervorgeht, da

1) Zeitschx. öffentl. Chem. 1904, 10, 284. 
ein solcher Zusatz vollkommen unnötig ist und nur dazu dienen kann, eine leichtere Zersetzbarkeit der Wurstmasse hervorzurufen ${ }^{1}$ ).

Bei den Brüh- und Rostbratwürsten wird aber von den Fleischern die Konzession eines geringen Mehlzusatzes gefordert. Diese Forderung wird allgemein, ganz abgesehen von etwa bestehenden Ortsgebräuchen aufgestellt und insbesondere durch die Angabe begründet, daß infolge der heutzutage üblichen $\mathrm{Schnellmast}$ das Fleisch bedeutend wasserhaltig, daher zur Wurstfabrikation nicht bindig genug und deshalb ungeeignet sei. Erst durch einen Zusatz von Mehl oder Eiweiß könne die Fleischmasse die richtige Bindigkeit wieder erlangen. Sofern sich die Angaben auf Ochsenund Kalbfleisch beziehen, sind sie jedenfalls unbegründet, denn es ist bekannt, daß zur Brühwurstfabrikation meist und am liebsten Bullenfleisch verwendet wird und daß Bullen keine schlechte Fütterung erhalten, darf wohl im Allgemeinen angenommen werden. Ähnlich dürfte es sich mit dem Kalbfleisch und schließlich auch mit dem Kuhfleisch verhalten, welches letztere vielfach anstatt des Bullenfleisches verwendet wird.

$O b$ bei Kühen übermäßig nasse Fütterung, insbesondere von Trebern, eine Verschlechterung des Fleisches für die Wurstfabrikation nach sich zieht, ist nicht erwiesen und jst in so allgemeiner Form auch höchst unwahrscheinlich. Dagegen hat die Klage der Fleischer inbezug auf das Schweinefleisch in gewissem Sinne seine Berechtigung, wie durch eine ganze Reibe von Gutachten der landwirtschaftlichen Verbände des deutschen Reiches und zusammenfassend in einem Gutachten des deutschen Landwirtschaftsrates zu Berlin vom 28. September 1905 zugegeben worden ist. Die gutachtlichen Äußerungen führen sich zurück auf eine Eingabe des deutschen Fleischerverbandes zu Frankfurt a. M. vom 3. März 1905 an den Reichskanzler, betreffend die "Verfälschung des Schweinefleisches bei der Mast", die eine Abänderung des \& 5 Ziffer 2 des Nahrungsmittelgesetzes fordert, dahingehend, daß bestimmte Arten der Fütterung, insbesondere von Olkuchen, Fischen, Fischabfällen, unentöltem Fischmehl, Fleisch kranker Tiere, rohem Centrifugenschlamm oder Blutmelasse durch reichsgesetzliche Regelung verboten werden solle. Es ist hier nicht der Ort, näher auf diese Gutachten einzugehen, soviel muß nur daraus hervorgehoben werden, daß die Klage insofern berechtigt erscheint, als der Landwirt jetzt vorzieht, mehr junge Schweine im Alter von 9-10 Monaten zu mästen, während früher die Mästung erst im 33. und 4. Lebensjahre begonnen wurde. Daß das Fleisch junger Tiere weniger fest als das älterer Tiere und daher zux Wurstfabrikation weniger geeignet ist, liegt auf der Hand. Es ist aber in diesem Verfahren der Mästung kein Betrug der Fleischer zu erblicken, weil sich diese die für ihre Zwecke geeigneten älteren Tiere aussuchen können. Andererseits wird auch zugegeben, dab die Fütterung von Ölkuchen das Fett der Schweine nachteilig beeinflußt, so zwar, daß dasselbe leichtflüssiger wird, was sich auch an der Erhöhung der Jodzahl zu erkennen gibt. Ähnlich wirken Fischmehl und Fischabfälle, die dem Fette auch einen tranigen Geruch rerleihen; desgleichen ist die Fütterung von Blutmelasse und Centrifugenschlamm aus hygienischen Gründen verwerflich; letzterer ist z. B. in Sachsen auch als Futtermittel gesetzlich verboten. Ist somit einerseits die Klage der Fleischer berechtigt, so kann andererseits doch ein in sich berechtigtes Zucht-Verfahren der Landwirte nicht als absichtliche Fälschung betrachtet werden und dasselbe fällt nicht unter das Nahrungsmittelgesetz, weil nach Entscheidungen des Oberlandesgerichts zu Kiel lebende

1) Vergl. Urteil des Reichsgerichts rom 23. September 1886 und des Kammergerichts vom 24. Januar 1901. 
Tiere nicht zu den Nabrungsmitteln zu rechnen sind. Diese ganze Frage läuft somit schließlich auf eine reine Geldfrage hinaus, da wohl zur Wurstfabrikation geeignetes Schweinefleisch auf dem Markte vorhanden ist, aber natürlich auch entsprechend teurer bezablt werden muß. $\mathrm{Ob}$ aber der Metzger sich ein für seine Zwecke geeignetes Fleisch beschaffen muß, und ob er sich daher den Marktpreisen anpassen muf, steht hier nicht zur Erörterung. Übrigens haben sich auch einzelne Fleischerinnungen, insbesondere die große Dresdener Fleischer-Innung, dahin ausgesprochen, daß das Fleisch, wie es auf dem Markte erhältich ist, zur Wurstfabrikation geeignet sei und dafo daher irgend welche Nachhilfemittel, insbesondere Mehl bei der Bereitung der hier nur in Betracht kommenden Brühwürstchen nicht nötig seien. Dagegen haben in manchen anderen Bezirken, auch neuerdings wieder in Leipzig und einigen sächsischen Amtshauptmannschaften, die Fleischerinnungen durchgesetzt, daßo ihnen ein Zusatz von $2 \%$ Mehl unter dem nötigen Deklarationszwang bewilligt wurde. An anderen Orten aber, wo man noch nicht so weit fortgeschritten ist, hat man dagegen seinen Blick auf das Eiweiß als Retter in der Not gerichtet und verwendet ein teils stärkefreies, teils stärkehaltiges Abfallprodukt der Stärkefabrikation, weil dasselbe „die Bindekraft der Wurst erhöht, ein saftiges Fabrikat liefert und allen gesetzlichen Anforderungen entspricht". Letzteres ist natürlich die Ansicht der Fabrikanten dieser Produkte und deren Agenten, also ein ähnliches Urtell, wie es von derselben Seite auch bei der Anpreisung der Benzoesäure vielfach bekannt ist. Es handelt sich nun um die Frage, ist ein solcher Zusatz nötig oder nicht, und welehen Einflub hat der Zusatz von Bindemitteln auf die Beschaffenheit und Zusammensetzung der Wurst? Unter Bindekraft versteht man bekanntlich die Fähigkeit des Fleisches zu quellen, so zwar, daß das Muskeleiweiß der Faser Wasser aufnimmat und eine Verbindung unter dem Wurstfüllsel herstellt. Die Bindung des Fleisches kann durch künstliche Mittel gesteigert werden, so bekanntlich durch Erwärmung oder Verarbeitung noch lebenswarmen Fleisches, wie es in Süddeutschland vielfach Sitte ist, dann aber auch durch Zusatz von Kalbfleisch, schließlich durch Traganth, Mehl oder EiweiB. Levztere Substanzen quellen in der Wärme unter Aufnahme von Wasser und stellen dadurch die Bindung her.

Es sind Versuche darüber angestellt worden, wie viel Wasser die Stärke zu binden vermag; so fand $K$ ohlman $n^{1}$, daß $60 \mathrm{~g}$ trockene Semmel mit Leichtigkeit $190 \mathrm{~g}$ Wasser, also das Dreifache ihres Gewichtes, aufzunehmen imstande sind; während Lintner ${ }^{2}$ ) angibt, daß Stärke 40\% Wasser zu binden vermag, ohne naß zu erscheinen. Diese Versuche sind an und für sich interessant, aber für die Beurteilung des Mehles bei der Brühwurstbereitung haben sie meines Erachtens wenig Wert, wenn man sich die Bereitung dieser Wurst vergegenwärtigt. Bullenfleisch und Schweinefleisch bezw. Speck werden unter Zusatz von Gewürzen und Salz und unter steigendem Wasserzusatz entweder mit der Hand gemengt oder durch den Kutter innig miteinander in Berührung gebracht. Dann wird das Gehäck in Därme (meist Schafsdärme) gespritzt, etwa $1 / 2$ Stunde in der Esse heißem Rauche ausgesetzt und schließlich $1 / 4-1 / 2$ Stunde in Wasser von $70^{\circ}$ gebrüht. Die Temperatur ist also nicht so hoch und die Einwirkung dauert nicht so lange, daß die Stärke viel Wasser zu binden vermag. Erst wenn die Wurst kräftig gekocht wird, dürfte mehr Wasser gebunden werden. Infolge

1) Zeitschr. öffentl. Chem. 1902, 8, 201.

^) Siehe König, Chemie d. Nahrungs- und Genußmittel. IV. Aufl, 2, 527. 
des Räucherns soll der Wassergehalt der Würstchen um ein bedeutendes sinken und die Wasseraufnahme dadurch unter Umständen ganz und gar kompensiert werden können.

Über die Frage, ob man bei Verwendung von Mehl mehr Wasser in die Wurst hinein zu kneten vermag, scheinen noch sehr verschiedene Ansichten zu herrschen. Nach dem vorher Gesagten ist eine erhöhte Bindung von Wasser bei meblhaltiger Wurst im allgemeinen nicht wahrscheinlich, da ja die Wasseraufnahme ein Quellprozeß ist und weil Mehl bei geringer Wärme nur wenig Wasser aufzunehmen vermag. Zu erwähnen sind in dieser Beziehung Versuche, die von Schorer und Küstermann') auf Veranlassung des Landgerichts zu Lübeck angestellt worden sind. Diese haben ein regelrechtes "Probewursten" angestellt und fanden dabei:

1. Zur Herstellung ordentlicher Brühwürstchen müssen auf 100 Teile Wurst etwa 18 Teile Wasser zugesetzt werden.

2. Beim Räuchern solcher Brühwürstchen verliert die Masse etwa $11 \%$ Wasser.

3. Ein Zusatz von $0,8 \%$ Stärke zur Fleischmasse ist obne wesentlichen Einfluf3 auf den Wassergehalt der Wurst; auch wird die äußere Erscheinung und der Geschmack der Wurst dabei nicht geändert; vorausgesetzt ist gut bindendes Fleisch.

4. Es lassen sich auf 100 Teile Fleisch bei $2 \%$ Mehlzusatz 70 Teile Wasser untermischen.

5. Auch ohne Mehlzusatz lassen sich 70 Teile Wasser auf 100 Teile Fleisch mischen.

6. Beim Räuchern verlieren die Würste mit $2{ }^{\circ} 0 \mathrm{Mehl}$ und die ohne Mehl an $n$ ähernd die gleiche Menge Wasser, und zwar 32 und 35 Teile.

Hieraus haben Schorer und $\mathrm{K}$ üstermann geschlossen:

a) Es läfat sich durch Kartoffelmehlzusatz von 2\%; keine größere Menge $W$ asser in die Wurst hineinarbeiten, als obne Kartoffelmehlzusatz möglich wäre.

b) Die Größe des Wasserverlustes beim Räuchern ist abhängig von dem Wasserzusatz, den die rohe Wurstmasse erfabren bat; derselbe wird nicht beeinflußt durch einen Kartoffelmehlgehalt, wenigstens nicht von $2 \%$.

Schon in Jahre 1887 sind von Trillich ${ }^{2}$ ) Versuche über den ,Wassergehalt mehlhaltiger Würste" angestellt worden, die das Ergebnis geliefert haben, daf man den Wassergehalt einer Wurst auch ohne Mehlzusatz auf jede beliebige Höhe bringen könnte, daß somit Mehlzusatz und Wassergehalt in keinem oder in einem nur nebensächlichen Zusammenhange ständen. In der neueren Literatur finden sich nur Arbeiten von v. Raumer auf diesem Gebiete, der zweimal über diesen Gegenstand berichtet hat. Er weist dabei in der ersten Arbeit ${ }^{3}$ ) auf die Trillich'schen Arbeiten hin und verurteilt sie. In der zweiten Arbeit ${ }^{4}$ ) über diesen Gegenstand, die sich insbesondere mit der wasserbindenden Kraft des Eiweißes und der sich daraus berechnenden Rentabilität des Eiweißzusatzes beschäftigt, setzte v. Raumer zu dem fertigen Wurstgehäck steigende Mengen Bindemittel und Wasser und erhielt bei Zusatz ron $1 \%$ Eiweiß und 20\% Wasser einen Mehrgewinn an Wurst von 19\%, bei $2 \%$ Eiweiß und $26 \%$ Wasser einen solchen von $26,7 \%$, bei $4 \%$ Eiweib und $40 \%$ Wasser einen solchen von $39,8 \%$. Daraus wird ein Mehrgewinn für $100 \mathrm{~kg}$ Wurst um 41,56 bezw. 54,80 und $75,52 \mathrm{Mk}$. berechnet.

1) Ostertag, Handbuch der Fleischbeschau 1902, S. 795 .

9) Trillich, Vierteljahresschrift ïber die Fortsehritte auf dem Gebiete der Chemie der Nahrungs- und Genufimittel, 1887, 2, 501.

$\left.{ }^{3}\right)$ Diese Zeitschrift 1905, 9, 405.

4) Diese Zeitschrift 1906, 11, 335 .

N. 07. 
Da ich mich mit der Frage der Wurstbindemittel, insbesondere dér Verwendung des Eiweißes, auf wiederholten Antrag von Gerichten eingehend zu beschäftigen hatte und in Gerichtsakten fast allgemein auch den Hinweis auf die v. Raumer'sche Arbeit fand, so beschloss ich zur Nachprüfung dieser Frage selbst praktische Versuche anzustellen, da mir durch diese 3 Versuche v. Raumer's doch die Frage nicht endgültig erledigt zu sein schien. Die Brühwürste wurden in der oben erwähnten, hier üblichen Weise hergestellt, nämlich aus Bullenfleisch, Schweinespeck, Salz, Pfeffer und Wasser, unter Zugabe steigender Mengen von Eiweiß (bezw. Mehl). Es wurde dem Gehäck soviel Wasser beigemischt, als es irgend zu fassen vermochte. Der Punkt der Überwässerung konnte leicht daran orkannt werden, daß das Fleisch schlüpfrig wurde und kleine weiße Bläschen deutlich wahrnehmbar waren. Es stellte sich dabei heraus, daß in alle Proben von demselben Gehäck gleich oder annähernd gleichviel Wasser in der Kälte hineingearbeitet werden konnte, ob nun viel, wenig oder gar kein Eiweib, oder schließlich Mehl zugefügt wurde. Die letzten beiden Sorten Würstchen wurden im Kutter gemischt, die ersteren mit der Hand gemengt. Die Wurstfabrikation geschieht hier so, daß das Rindfleisch mit Salz und Mehl in der Mulde bestreut und allmählich Wasser eventuell unter Bindemittelzusatz hineingearbeitet wird. Nachträglich wird erst der Schweinespeck oder das fette Schweinefleisch hineingeknetet. Die Würstchen wurden genau wie üblich gespritzt, geräuchert und gebrüht, dann erkalten gelassen und am darauffolgenden Tage auf ihren Wassergehalt untersucht. Ich füge hinzu, daß ich der Fabrikation von Anfang bis zum Ende beiwohnte. Das Räuchern dauerte etwa $1 / 2$ Stunde, das Brühen $1 / 4-1 / 2$ Stunde. Bei den Versuchen No. 8 und 9 wurde etwas länger geräuchert. In der nachfolgenden Tabelle sind die Versuchsergebnisse zusammengestellt, wobei noch zu bemerken ist, daß der Wassergehalt der Wurst infolge der nicht ganz gleichmäbigen Verteilung des Fettes in der Wurst bei doppelten Bestimmungen stets gewissen Schwankungen unterliegt, zumal wenn, wie im vorliegenden Falle, die Bestimmung zur Kontrolle in 2 verschiedenen, aber gleichzeitig bereiteten Würstchen vorgenormmen wird.

\begin{tabular}{|c|c|c|c|c|c|c|c|c|}
\hline \multirow{2}{*}{$\begin{array}{l}\text { Ver- } \\
\text { such } \\
\text { No. }\end{array}$} & \multicolumn{5}{|c|}{ Angewendet $\ominus$ Mengen } & \multicolumn{3}{|c|}{ Wassergehalt der Wurst } \\
\hline & Fleiseh & Koehsalz & Pfeffer & $\begin{array}{l}\text { Eiweib bei der } \\
\text { Ferarbeitung }\end{array}$ & Wasser & I & II & Mittel \\
\hline 1 & $1500 \mathrm{~g}$ & $26 \mathrm{~g}$ & $10 \mathrm{~g}$ & 0 & 0 & $62,03 \%$ & $63,60 \%$ & $62,79^{\circ}$ \\
\hline 2 & $1500 \%$ & $26 n$ & 10 & 0 & $450 \mathrm{~g}$ & 65,31 & 64,82 , & 65,07 \\
\hline 3 & 1500 & 26 n & 10. & $15 \mathrm{~g}$ & 450 & 66,39 , & 67,69 & 67,04 , \\
\hline 4 & $1500 \%$ & 26 & 10 & 30 & 585 & 66,52 & 62,91 & 64,72 \\
\hline 5 & 1500 * & 26 & 10 & $\begin{array}{c}75, \\
\text { Tierisches }\end{array}$ & 500 & 65,12, & 64,58 & 64,85 , \\
\hline 6 & 1250 & $26 \pi$ & 10 & $\begin{array}{c}\text { Eiweil } \\
75 \mathrm{~g} \\
\text { Kartoffelmebl }\end{array}$ & 500 & 63,38 & 62,57 & 62,98 " \\
\hline 7 & 1250 & $26 n$ & 10 & $\begin{array}{c}75 \mathrm{~g} \\
\text { Eiweib }\end{array}$ & 400 & 61,05 & - & 61,05, \\
\hline $\begin{array}{l}8 \\
9\end{array}$ & $\begin{array}{l}1500 \% \\
1500\end{array}$ & $\begin{array}{l}30 \\
30\end{array}$ & $\begin{array}{l}10 \\
10\end{array}$ & $\begin{array}{c}30 \mathrm{~g} \\
0\end{array}$ & $\begin{array}{l}200 " \\
200 "\end{array}$ & $\begin{array}{l}55,07 \\
55,20\end{array}$ & - & $\begin{array}{l}55,07 " \\
55,20 "\end{array}$ \\
\hline
\end{tabular}

Bei Versuch No. 6 wurde tierisches Eiweiß (Handelspräparat), bei No. 7 Kartoffelmehl, in allen übrigen Fällen Eiweiß mit etwa $90 \%$ Stickstoff-Substanz verwendet. Gleichzeitig wurde der Wassergehalt der Schweinespeckproben und des verwendeten Fleisches bestimmt. Der erste betrug etwa 10 bezw. $8^{\%} \%$, der des Fleisches etwa $75 \%$ bei 
den ersten 7 Versuchen, bei No. 8 und 9 aber etwa $70 \%$. Das erstere Fleisch war rom Fleischer dem Aussehen nach als nicht bindig bezeichnet worden und wurde daher gerade aus diesem Grunde für die Versuche ausgewählt. Die Versuche No. 8 und 9 sind demnach als Gegenversuche mit bindigem Fleische aufzufassen. Das angeblich nicht bindige Fleisch (Bullenfleisch) war also wasserreicher, doch konnte ich bei der Verarbeitung zur Wurst eine Minderwertigkeit des Fleisehes gegenüber dem bei 8 und 9 verwendeten Fleische nicht erkennen.

Die Ergebnisse der Wasserbestimmungen zeigen, daß ein wesentlicher Unterschied im Wassergehalt der mit und ohne Bindemittel verarbeiteten. Würste nicht vorhanden ist. Nur bei den letzten beiden maschinell verarbeiteten Würsten ist der Wassergehalt etwas erniedrigt. Ein richtiges Bild über die Zusammensetzung der Wurst ergibt die nachfolgende Tabelle, welche die Verwendung der Materialien in Prozenten aufführt:

\begin{tabular}{|c|c|c|c|c|c|c|}
\hline \multirow{2}{*}{ No, } & \multirow{2}{*}{$\begin{array}{c}\text { Wasser im } \\
\text { Bullen- } \\
\text { fleisch }\end{array}$} & \multirow{2}{*}{$\begin{array}{l}\text { Wasser im } \\
\text { Sehweine- } \\
\text { fleisch }\end{array}$} & \multirow{2}{*}{\begin{tabular}{|c|} 
Zugesetztes \\
Wasser
\end{tabular}} & \multicolumn{2}{|c|}{$\begin{array}{l}\text { Wassergehalt } \\
\text { der Wurstmasse }\end{array}$} & \multirow{2}{*}{$\begin{array}{c}\text { Bindemittelzusatz auf die gesamte } \\
\text { Wurstmasse berochnet }\end{array}$} \\
\hline & & & & berechnet & gefunden & \\
\hline 1 & $75 \%$ & $10 \%$ & 0 & $58 \%$ & $62,79 \%$ & 0 \\
\hline 2 & $75 n$ & 10 & $23 \%$ & 65 & 65,07 & 0 \\
\hline 3 & 75 & 10 & 23 & 64 & 67,04 & $0,4 \%$ Eiweiß ${ }^{\circ}$ Althen ${ }^{*}$ \\
\hline 4 & 75 & 10 & 27 n & 65 , & 64,72 & 0,8 \\
\hline 5 & 75 & $10 n$ & $24 \%$ & 64 & 64,85 & 2,0 \\
\hline 6 & $75 \%$ & 10 & 28 & 62 & 62,98 & 3,1, Tierisches Eiweiß \\
\hline 7 & $75 ”$ & $10 "$ & 22 & 61 & 61,05 & 3,3 \# Kartoffelmehl \\
\hline 8 & 70 & 8 & $9 "$ & 57 & 55,07 & etwa $10_{0}$ Eiweif ,Althen" \\
\hline 9 & 70 & $8 \pi$ & 9 & 58 & 55,20 & 0 \\
\hline
\end{tabular}

Aus dieser Tabelle ersehen wir zunächst, daß ein Wasserverlust beim Räuchern und Brühen nicht stattgefunden hat, daB im Gegenteil bei den Würsten, welche weder Wasser- noch Eiweißzusatz erfahren hatten (No. 1), der Wassergehalt, offenbar beim Brühen, um etwa 5\% gestiegen ist. Auch bei Probe No.3 ist der Wassergehalt ein erböhter und ist die Ursache hierfür nicht nachgewiesen worden. Bei den übrigen, aus angeblich "nicht bindigem" Fleische hergestellten Würsten war der gefundene Wassergehalt mit dem berechneten gleich. Nur bei den aus „bindigem“ Fleische vermittels der Maschine hergestellten Würsten (No. 8 und No. 9) war der Wassergehalt um 2-3\% gesunken. Da die Würste regelrecht hergestellt waren, so zeigen die Versuche, daß im vorliegenden Falle von einer Wasseraufnahme infolge der Beimischung von Eiweiß nicht gesprochen werden kann. Die Versuche No. 7, 8 und 9 lassen dagegen erkennen, daß mit einer Herabsetzung des Wasserzusatzes auch der Wassergehalt der fertigen Wurst zurückgeht. Das erklärt aber auch gleichzeitig, daß, wenn man steigende Mengen Wasser und Bindemittel zusetzt, man auch entsprechend steigende Mengen Wasser in der Wurst wiederfinden wird, wie v. Raumer bewiesen hat. Man kann aber dabei die Bindemittel auch fortlassen, ohne das Ergebnis zu beeinträchtigen. Andererseits ist diese Frage des Wassergehaltes bei Brühwürstehen nur dann durch Versuche einwandfrei zu erledigen, wenn man gleiche Mengen Wasser und steigende Mengen Bindemittel verwendet, da sonst komplizierte Terhältnisse eintreten. 
Übrigens kommt auch der Wassergehalt bei Brühwürstchen nicht so sehr in Frage, da diese nicht nach dem Gewichte, sondern nach der Zahl verkauft werden. Da die Würste nach Gutdünken abgebunden werden, so kann ernstlich von einer Erhöhung des Wassergehaltes nicht geredet werden. Anders ist es bei Dawerwürsten, doch ist der Standpunkt, der den Mehlzusatz bei solehen charakterisiert, sehon oben dargelegt worden. Ich muß noch erwähnen, daß bei einer anderen Wurstbereitungsart etwas andere Ergebnisse wohl hätten erhalten werden können und daß insbesondere der Räucherprozeß eine Verringerung des Wassergehaltes hätte herbeiführen können. Leider habe ich vorläufig keine Gelegenheit, weitere Versuche in dieser Richtung anzustellen. Der Wassergehalt der Würste ist viel mehr von anderen Faktoren abhängig als vom Eiweiß, also vor allen Dingen von der Art und dem Wassergehalte des Fleisches und des Specks, vom Salz- und Gewürzzusatz, von der Art des verwendeten Darmes, von der Fabrikation, von der Länge des Räucherns und Brühens usw. Für alle Fälle gültige Versuche anzustellen, dürfte sehr schwer sein, wie ja aus den sich fortgesetzt widersprechenden Berichten über solche Versuche hervorgeht, und man ist nicht einmal zu dem Schlusse berechtigt, daß mit einem Zusatz von Eiweiß oft ein erhöhter Wassergehalt der Wurst Hand in Hand geht. Die Praxis allein bietet schließlich die Möglichkeit, über solche Fragen zu entscheiden und die Praxis hat im vorliegendem Falle gezeigt, daß mehlhaltige Wurst durchaus nicht immer oder auch nur durchgängig einen höheren Wassergehalt aufweist. So fand Röhrigl), daß von 20 Wurstproben die Brühwürste mit dem höchsten Wassergehalt keine Stärke enthielten und saftiger waren als die mehlhaltigen Würste. Die im chemischen Untersuchungsamte zu Chemnitz im Jahre 1906 in dieser Richtung gemachten Beobachtungen gingen dahin, daß $44 \mathrm{mehlhaltige} \mathrm{Brähwürste} \mathrm{folgenden} \mathrm{Gehalt} \mathrm{an}$ Wasser aufwiesen:

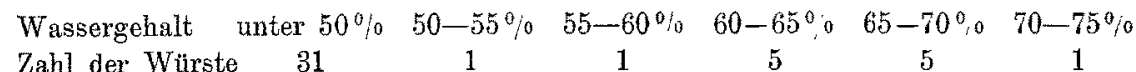

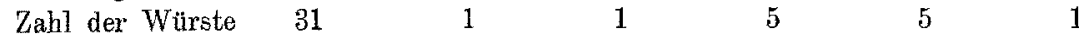

Bei einer Wurst betrug der Wassergehalt $70,60 \%$ bei $3,96 \%$ Stärke und bei einer anderen $48,37 \%$ bei $3,58 \%$ Stärke.

Bei 52 mehlfreien Würsten dagegen schwankte der Wassergehalt innerhalb folgender Grenzen:

$\begin{array}{lcccccc}\text { Wassergehalt unter } 50 \% & 50-55 \% & 55-60 \% & 60-65 \% & 65-70 \% & 70-75 \% \% \\ \text { Zahl der Würste } & 7 & 3 & 3 & 14 & 17 & 8\end{array}$

Diese Zahlen sprechen für sich selbst. Auch hier kann von einem höheren Wassergehalt der mehlhaltigen Würste nicht wohl geredet werden, dagegen zeigen die mehlfreien Würste z. Tl. recht erhebliche Wassermengen.

Von einer ganz anderen und weit wichtigeren Seite dürfte vielmehr der Bindemittelzusatz zur Wurst zu betrachten und zu beurteilen sein. Die natürliche Bindekraft frischen Fleisches verbietet die Verwendung von Eiweißbindemitteln bei der Wurstfabrikation wegen deren meist unverbältnismäBig hohen Kaufpreises ganz von selbst, dagegen ist ein solcher Zusatz zur Wiedergewinnung der Bindekraft vorteilhaft. Es mag wohl vorkommen, dab verdorbenes Fleisch und Wurstreste zur Wurstfabrikation wieder verwendet werden, doch ist ein solches Verfahren nicht so allgemein verbreitet, daß es als durchgängig bei der Beurteilung herangezogen werden könnte.

3) Röhrig, Bericht des chemischen Untersuchungsamtes der Stadt Leipzig 1904, S. 20. 
Aber die Verwendung von geringeren Qualitäten Fleisch und Fleischabfällen zur Wurstbereitung würde durch die Freigabe von $W$ urstbindemitteln wesentlich gefördert werden. Das Publikum muß aber verlangen, daß nicht Fleisch geringster Qualität, z. B. sehniges Fleisch des Beckens und des Bauches zur Wurstfabrikation verwendet wird, dessen natürliche Bindekraft eine geringe ist, die aber durch Anwendung von Bindemitteln, insbesondere auch von Eiweib, erhöht werden kann. Zwecks besserer Verwendung weniger bindigen $\mathrm{Rindfleisches} \mathrm{-} \mathrm{falls} \mathrm{solches} \mathrm{überhaupt} \mathrm{existiert} \mathrm{-} \mathrm{zur}$ Wurstfabrikation, gibt es andere einwandfreie Mittel, die ich oben schon erwähnt habe, nämlich den Zusatz von Kalbfleisch oder Erwärmen. Auf dem Lande und in Privathäusern ist es bisher noch nicht Brauch gewesen, Wurst unter Verwendung von Mehl oder Eiweiß herzustellen und es ist kein Grund einzusehen, warum die Fleischer eine solche Manipulation anzunehmen brauchen, die schließlich ein Gewohnheitsrecht nicht nur in dieser Finsicht werden würde, sondern auch von anderen Nahrungsmittelzweigen als Präzedenzfall gebraucht werden könnte. Verwenden doch große Fleischereien grundsätzlich keine Bindemittel und beweisen dadurch, daß bei Verwendung guter Rohstoffe solche nicht nötig sind. Gerade aber beim Fleischergewerbe, dem wichtigsten Gewerbe der Nahrungsmittelindustrie, ist es nötig, daß auf die besten Rohstoffe gehalten wird und das liegt im eigensten Interesse der Fleischer selbst. Dem Mehlzusatze konnte man leichter zu Leibe gehen, weil Kartoffelmehl oder Semmel eine im Verhältnis zum Fleisch minderwertige Substanz genannt werden muß und daher direkt als eine Verfälschung anzusehen war. Beim Eiweib ist das etwas anderes, denn dieses verringert den Nährwert des Fleisches nicht wesentlich. In wiederholten Urteilen des Reichsgerichts ist aber dahin entschieden worden, daß „eine Verfälschung im Sinne des $\$ 10$ des Nahrungsmittel-Gesetzes nicht bloß im Wege einer Verschlechterung der Beschaffenheit, sondern auch dadurch herbeigeführt werden kann, daß dem Nahrungsmittel der Schein einer besseren Beschaffenheit als der in Wahrheit vorhandenen verliehen wird". Das Eiweiß soll aber dazu dienen, die verloren gegangene oder nicht vorhandene Bindekraft des Fleisches künstlich zu ersetzen. Die Würste, die unter Verwendung von Eiweiß hergestellt sind, zeigen demnach eine bessere Beschaffenheit, als sie ohne den Zusatz dieses Mittels normalerweise haben würden.

Im Interesse des reellen Handels muß man daher folgerichtig gegen alle Bindemittel, sie mögen heißen wie sie wollen — und das Eiweiß wird sicherlich bald durch ein anderes abgelöst werden - vorgehen. Daß aber eine Einheitlichkeit auf diesem Gebiet nicht so bald erzielt werden wird, darüber braucht man sich keinen Illusionen hinzugeben.

In der praktischen Nahrungsmittelkontrolle ist die Beurteilung der Bindemittel augenblicklich eine akute Tagesfrage, die um so mehr Aufmerksamkeit verlangt, als unser Standpunkt in dieser Frage von den Fleischern sofort als ein prinzipieller ausgebeutet werden wird. Zweck dieser Arbeit war demnach, die über diesen Gegenstand erschienenen wichtigsten Arbeiten zusammenzufassen, wobei festgestellt werden kann, då, wenn auch über die Wirkungsweise der Bindemittel entgegenstehende Auffassungen vorhanden sind, man schließlich doch in der Beurteilung und Verurteilung dieses „Handelsbrauches" einig ist.

Februar 1907. 